\title{
Photocoagulation treatment for clinically significant radiation macular oedema
}

\author{
James L Kinyoun, Ronald W Zamber, Betty S Lawrence, William E Barlow, \\ Alice M Arnold
}

\begin{abstract}
Macular oedema is a leading cause of vision loss in patients with radiation retinopathy. In an effort to find an effective treatment for this vision threatening complication, 12 eyes (eight patients) were treated with photocoagulation for clinically significant radiation macular oedema (CSRMO) defined as central macular thickening, exudates threatening the macular centre, or one disc area of thickening in the macula. Median visual acuity improved from $20 / 100$ preoperatively to $20 / 90$ at the initial postoperative examination (mean follow up 5 months) and to $20 / 75$ at the final postoperative examination (mean follow up 39 months). At the final postoperative examination, visual acuity had improved in eight $(67 \%)$ eyes and six $(50 \%)$ eyes had complete resolution of the CSRMO; two $(17 \%)$ other eyes had improved anatomically in that fewer CSRMO criteria were present. These results suggest that macular photocoagulation is effective in decreasing macular oedema and improving vision in eyes with CSRMO.

(Brf Ophthalmol 1995; 79: 144-149)
\end{abstract}

Radiation is being used with increasing frequency to treat not only malignant but also benign orbital, ocular, head, and neck tumours. Despite improved localisation and increased precision of dosage calculation and delivery, normal surrounding tissue continues to be adversely affected. Particularly sensitive to harmful effects of radiation are the capillaries supplying the macula; the resultant radiation macular oedema is a frequent cause of vision loss in patients after radiation treatment. $^{12}$ If the Collaborative Ocular Melanoma Study $^{3}$ results show that radiation treatment is preferable to enucleation, it will be even more imperative to find effective treatment for secondary complications of the radiation treatment, especially radiation macular oedema.

Short term results of one such treatment, photocoagulation, for clinically significant radiation macular oedema (CSRMO) in four patients (five eyes) have previously been published. ${ }^{4}$ We now report longer follow up findings in these patients plus the visual acuity and anatomical results of treatment in four additional patients (seven eyes).
Patients and methods

Eight patients, four with bilateral involvement or a total of 12 eyes, were enrolled and gave informed consent for macular photocoagulation treatment. To be eligible, affected patients had to have a history of radiation treatment around the eyes or head as well as clinical findings consistent with radiation retinopathy (microaneurysms, telangiectasia, and/or nerve fibre layer infarcts) plus radiation macular oedema with one or more criteria for being clinically significant as defined by the Early Treatment Diabetic Retinopathy Study Research Group. ${ }^{5}$ These criteria include:

(1) (centre) retinal thickening at or within $500 \mu \mathrm{m}$ of the centre of the macula;

(2) (exudates) hard exudates within $500 \mu \mathrm{m}$ of the centre of the macula, if associated with adjacent retinal thickening; or

(3) (zone) a zone or zones of retinal thickening one disc area or larger, any part of which is within one disc diameter of the centre of the macula.

Eyes with macular oedema which was not clinically significant (retinal thickening or appreciable hard exudates within one disc diameter of the centre of the macula) ${ }^{6}$ were not eligible for treatment. None of our patients' retinopathy was suggestive of a branch vein occlusion (sectoral distribution) or central retinal vein occlusion (venous dilatation). One patient had adult onset diabetes mellitus but the retinopathy did not occur until 11 months after radioactive plaque treatment, and this patient's opposite macula was normal. Examination techniques used to detect radiation retinopathy, macular oedema, and CSRMO included ophthalmoscopy (direct and indirect), contact lens fundus biomicroscopy, and stereoscopic colour fundus photographs.

Snellen visual acuity was measured and recorded by independent examiners at the preoperative and postoperative examinations. For eyes with visual acuity less than $20 / 400$, acuity was recorded as counting fingers or hand movements. For visual acuity analyses, counting fingers and hand movements were arbitrarily converted to $20 / 800$ and $20 / 1600$, respectively. Preoperative stereoscopic colour fundus photographs and fluorescein angiograms were obtained for all 12 study eyes. CSRMO criteria were recorded for the preoperative examination, the postoperative examination occurring closest to 4 months following treatment (referred to as initial \\ 27 September 1994
}




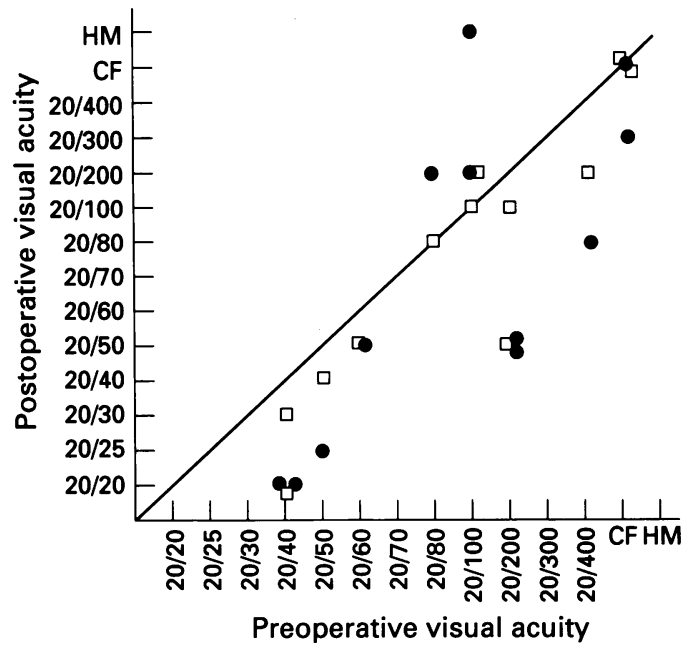

Figure 1 The squares (initial postoperative examination) and circles (final postoperative examination) below the line represent the eyes with improved vision when comparing the preoperative visual acuity with initial and final postoperative visual acuities. The symbols above the line represent the eyes with worse visual acuity, and the symbols on the line represent eyes with the same visual acuity pre- and postoperatively. ( $C F=$ counting fingers; HM = hand movements.)

postoperative examination), and the final postoperative examination. Because of sample size, it is not likely that any comparisons are statistically significant; therefore, we limit ourselves to descriptive statistics.

The photocoagulation treatment techniques used in our patients are the same as those recommended for treatment of clinically significant diabetic macular oedema. ${ }^{57}$ Fluorescein angiograms were used to identify leaking microaneurysms and areas of capillary non-perfusion and diffuse leakage judged to be responsible for the CSRMO. Fifty and $100 \mu \mathrm{m}$ green and blue green laser burns were used to turn the microaneurysms dark red or white; the same size burns of moderate intensity and spaced one burn diameter apart were used when applying a limited scatter (grid) treatment to areas of capillary non-perfusion and diffuse leakage.

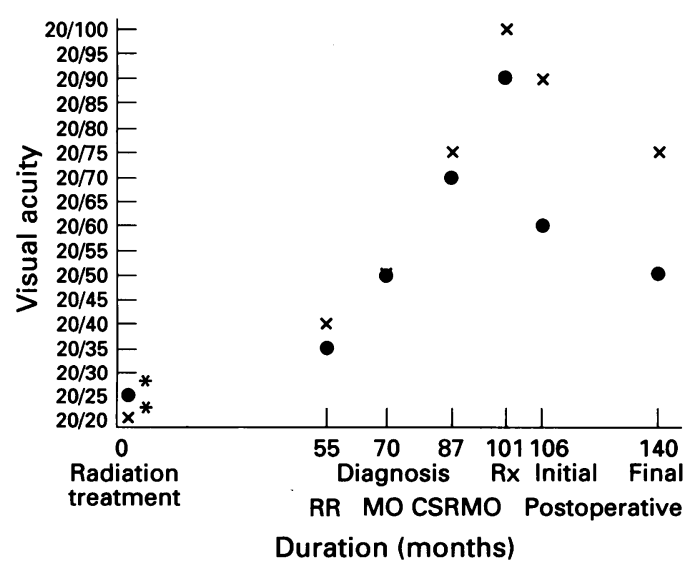

Figure 2 The $x$ axis represents scaled time (mean months) for each recorded visual acuity [mean ( $\bullet$ ) and median $(X)]$ on the $y$ axis. ${ }^{\star}$ Data for eight eyes. $R R=$ radiation retinopathy; $M O=$ macular oedema; $C S R M O=$ clinically significant radiation macular oedema; $R X=$ macular photocoagulation.

\section{Results}

Five men and three women were enrolled. Seven right eyes and five left eyes met the inclusion criteria for macular photocoagulation treatment. The four eyes in these patients which were not treated included two normal eyes of two patients with unilateral intraocular tumour, one eye with a markedly atrophic nerve and macula due to radiation damage, and one eye with very early radiation retinopathy (only occasional retinal haemorrhages and microaneurysms). The mean age of these patients at the time of radiation treatment was 35 years with a range of 6 months to 62 years. The mean age at the first treatment for CSRMO was 44 years with a range of 17 to 70 years. The indications for radiation treatment included three patients (five eyes) with Graves' ophthalmopathy, ${ }^{8}$ three patients (five eyes) with central nervous system tumour, and two patients (two eyes) with intraocular tumour. Eleven eyes were treated with external beam irradiation and one eye had local radioactive plaque treatment of a peripheral choroidal melanoma. Mean fraction size was $276 \mathrm{rad}$ based on a mean total of $4971 \mathrm{rad}$ given over a mean total of 18 fractions (ranges of 2000 to $10710 \mathrm{rad}$ and 1 to 33 fractions). The mean (median) latencies for the first diagnosis of radiation retinopathy, macular oedema, and CSRMO were 55 (27), 70 (34), and 87 (83) months, respectively. The range for each of these three diagnoses was 11-273, 11-273, and 11-329 months, respectively.

At the time of macular photocoagulation, all 12 eyes had thickening involving the centre of the macula, seven eyes had hard exudates associated with thickening, and 10 eyes had a disc area of thickening. The median preoperative visual acuity for these 12 eyes was $20 / 100$ with a range of $20 / 40$ to counting fingers. At the initial postoperative examination (mean of 5 months with range of 3-8 months after treatment), the median visual acuity was $20 / 90$ with a range of $20 / 20$ to counting fingers. The mean total follow up for these 12 eyes was 39 (range 6-76) months. Median visual acuity at the final postoperative examination was $20 / 75$ with a range of 20/20 to hand motion.

During follow up, these 12 eyes had an average of one retreatment for persistent or recurrent clinical significant macular oedema. The total number of retreatments ranged from 0 to 4; eight eyes had one or no retreatment. Additional macular photocoagulation was recommended for two patients (two eyes) at the final postoperative examination.

We found that a majority of eyes (seven and eight) had better visual acuity at both the initial and final postoperative examinations, respectively (Fig 1). Four eyes had at least halving of the visual angle at the initial postoperative examination and seven eyes had improved this amount at the final postoperative examination. One and three eyes at the initial and final postoperative examinations, respectively, had worse vision. Four eyes had the same vision at the initial postoperative examination and one eye had the same vision at the final examination. The mean total follow up for the eyes 


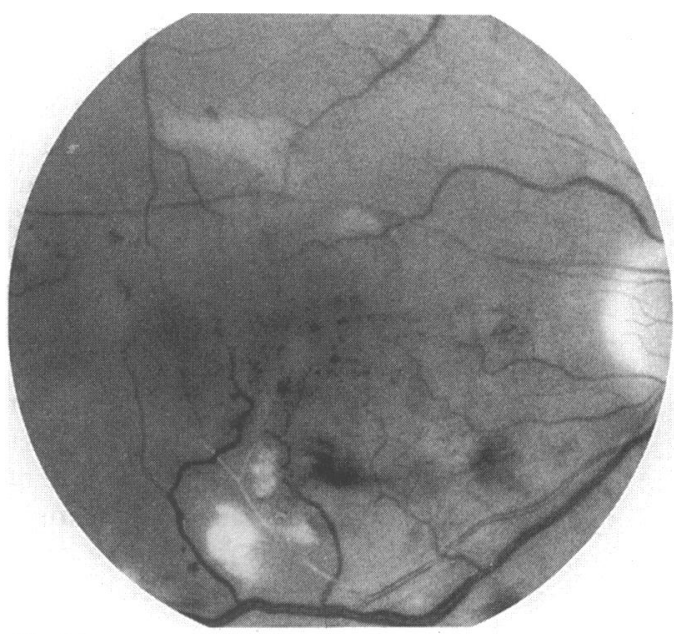

Fig $3 A$

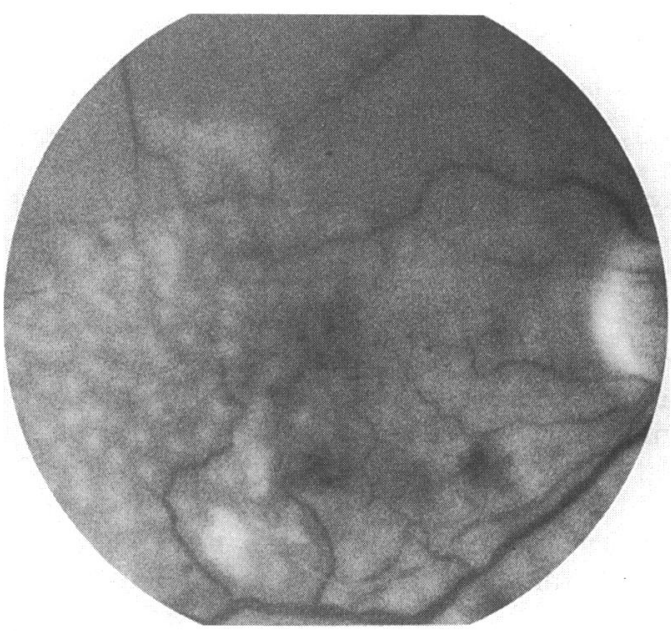

Fig $3 C$

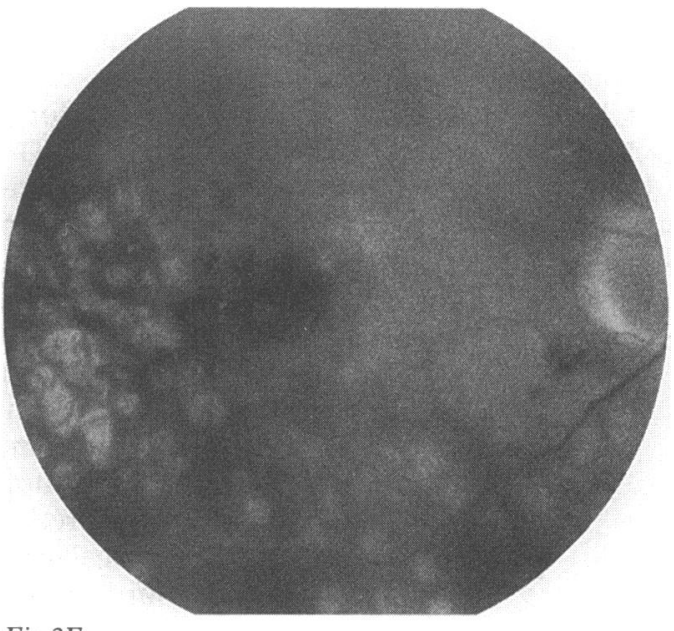

Fig $3 E$

with better and worse vision was 34 and 43 months, respectively The one eye with the same vision had been followed for 65 months.

The mean latencies and median and mean visual acuities of these patients are graphically summarised in Figure 2. Note that visual acuity measurements before radiation treatment are not available for four of these 12 eyes but were available for all 12 eyes before macular photocoagulation. Visual acuity progressively decreased following radiation treatment until macular photocoagulation was applied at a mean follow up of 101 months after radiation treatment and 31 and 14

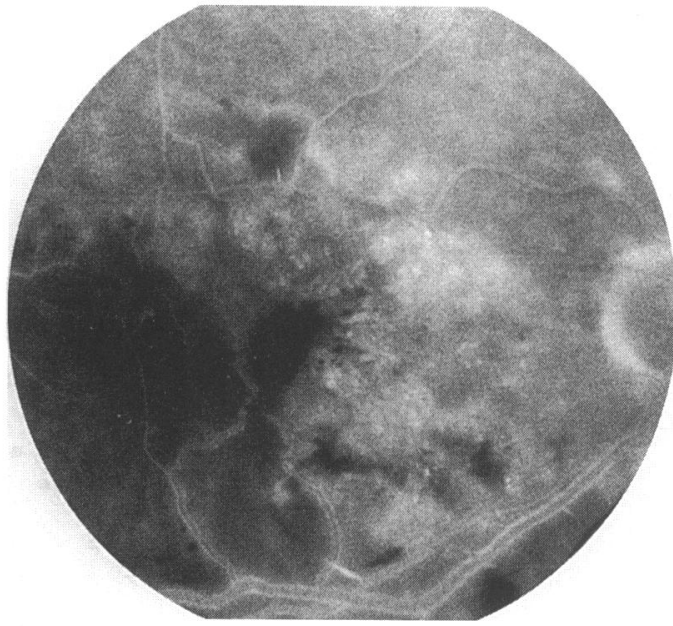

Fig 3B

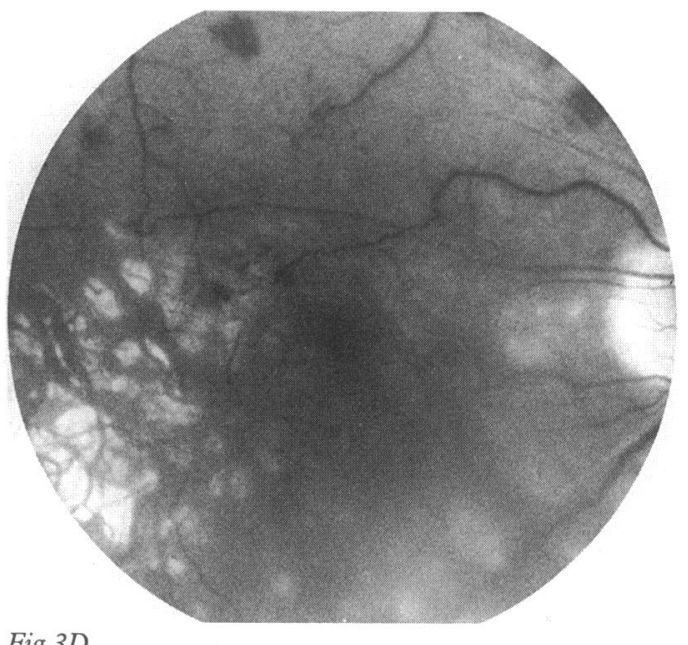

Fig 3D

Figure 3 (A) Preoperative fundus photograph with more than one disc area of retinal thickening which involves the central and inferior macular (CSRMO criteria 1 and 3).

Nerve fibre layer infarcts are present but hard exudates are absent (CSRMO criterion 2 is not present).

(B) Preoperative fluorescein angiogram (late phase)

demonstrates leakage of dye, capillary non-perfusion, and staining of vessel walls. (C) Fundus photograph immediately after treatment shows limited scatter photocoagulation burns. (D) Final postoperative fundus photograph shows photocoagulation scars and decreased nerve fibre layer infarcts. CSRMO criteria 1 and 3 are no longer present (compared with $(A)$ ). The media haze is due to two confounding factors (vitreous haemorrhage and posterior subcapsular cataract). (E) Final postoperative fluorescein angiogram showing hyper-and hypofluorescence of the photocoagulation scars, decreased leakage of dye, and absence of vascular wall staining (compared with (B)).

months, respectively, after the first diagnosis of macular oedema and CSRMO.

The total number of CSRMO criteria at the time of the preoperative, initial postoperative, and final postoperative examinations are summarised in Table 1 . Six eyes did not have any criteria for CSRMO at the final postoperative examination. Five and six eyes preoperatively had two and three total criteria, respectively; approximately half as many eyes had these total criteria at the final postoperative examination. Also, the percentage of eyes with each criterion decreased following photocoagulation treatment, from $100 \%$ for 
Table 1 Clinically significant radiation macular oedema

\begin{tabular}{llll}
\hline & \multicolumn{3}{l}{ Eyes (\%) } \\
\cline { 2 - 4 } Total \\
\cline { 2 - 4 } criteria & Preoperative & Postoperative & \\
\cline { 2 - 4 } & 0 & $2(17)$ & Final \\
\hline 0 & $1(8)$ & $1(8)$ & $6(50)$ \\
1 & $5(42)$ & $6(50)$ & $2(17)$ \\
2 & $6(50)$ & $3(25)$ & $3(25)$ \\
3 & & & \\
\hline
\end{tabular}

Table 2 Confounding factors

\begin{tabular}{llll}
\hline & \multicolumn{3}{l}{ Final postoperative visual acuity of eyes (\%) } \\
\cline { 2 - 4 } Factor (number of eyes affected) & Better & Same & Worse \\
\hline Macular ischaemia (12) & $8(67)$ & 1 & 3 \\
Optic neuropathy (7) & $3(43)$ & 1 & 3 \\
Panretinal photocoagulation (6) & $2(33)$ & 1 & 3 \\
Vitreous haemorrhage (2) & $1(50)$ & 0 & 1 \\
Cataract (2) & $1(50)$ & 0 & 1 \\
Repaired traction macular detachment & 0 & 0 & 1
\end{tabular}

criterion one at the preoperative examination to $75 \%$ and $42 \%$ at the initial and final postoperative examination, respectively. For criteria two and three, the percentages decreased from $58 \%$ to $50 \%$ and $33 \%$ and from $83 \%$ to $58 \%$ and $42 \%$, respectively (Figs 3 and 4).

Confounding factors which affected the analysis of visual acuity and anatomical results include macular ischaemia (parafoveal capillary dropout) in all 12 eyes, radiation optic neuropathy (based on disc pallor and visual field abnormalities) in seven eyes, prior panretinal photocoagulation in six eyes, vitreous haemorrhage in two eyes, cataract in two eyes, and a repaired traction macular detachment in one eye. The one eye with a repaired traction retinal detachment had worse vision at the final postoperative examination. Of the other five confounding factors, panretinal photocoagulation was associated with the lowest percentage of eyes with better vision at the final postoperative examination (Table 2). Three of the four eyes with four confounding factors had worse vision at the final postoperative examination (Table 3). Of the six eyes with no CSRMO criteria at the final postoperative examination, four had better vision; the other two eyes had four confounding factors and worse vision.

\section{Discussion}

Vision loss as a complication of radiation treatment designed to preserve vision is particularly undesirable. If radiotherapy complications cannot be prevented, it behoves physicians to find efficacious means for treating the complications. We believe these long term visual acuity and anatomical results of photocoagulation for CSRMO suggest that laser treatment is beneficial and not harmful for this sight threatening complication. We agree with others ${ }^{1}$ that spontaneous improvement in radiation macular oedema is unlikely to occur, and the experience of other investigators also indicates that photocoagulation treatment of radiation macular oedema is beneficial. ${ }^{9}$ The effectiveness of photocoagulation, however, will remain uncertain until a controlled, randomised treatment trial is conducted. This study provides useful pilot data for design of such a trial.

The visual and anatomical results in our patients may have been better if the patients had been treated earlier in their disease process. The diagnosis of CSRMO was not made until an average of over 7 years after the diagnosis of radiation retinopathy. More than a year elapsed, on average, before the CSRMO was photocoagulated. It is likely that photocoagulation treatment, as is the case with diabetic macular oedema, ${ }^{5}$ is more effective in preventing vision loss than improving vision already lost due to radiation macular oedema. Three eyes (cases 2,5 , and 8 ) had good visual acuity (20/20 to $20 / 40)$ at the final postoperative examination, and these three also had the best preoperative visual acuity (20/40 to $20 / 50$ ) of the 12 study eyes (Table 3 ).

The hallmark clinical finding of radiation retinopathy is the widespread capillary nonperfusion demonstrated with fluorescein angiography. The resultant macular ischaemia (defined as capillary dropout around the fovea) seen in all 12 of our study eyes supports present knowledge that the primary pathology in radiation retinopathy is damage to vascular endothelial cells. ${ }^{10}$ Capillary non-perfusion also occurs in diabetic retinopathy but usually not so extensively as seen in radiation retinopathy. The more extensive capillary non-perfusion in radiation retinopathy may decrease the beneficial effect of photocoagulation treatment for radiation macular oedema, but the extent of capillary non-perfusion necessary to eliminate any possible beneficial effect is not known

Table 3 Summary of study data (12 eyes)

\begin{tabular}{|c|c|c|c|c|c|c|c|c|c|c|}
\hline \multirow[b]{2}{*}{ Case } & \multirow[b]{2}{*}{ Eye } & \multicolumn{2}{|c|}{ Preoperative } & \multicolumn{3}{|c|}{ Initial postoperative } & \multicolumn{3}{|c|}{ Final postoperative } & \multirow[b]{2}{*}{ Confounderst } \\
\hline & & $\begin{array}{l}\text { Visual } \\
\text { acuity }\end{array}$ & $\begin{array}{l}\text { CSRMO } \\
\text { criteria }^{\star}\end{array}$ & $\begin{array}{l}\text { Follow up } \\
\text { (months) }\end{array}$ & $\begin{array}{l}\text { Visual } \\
\text { acuity }\end{array}$ & $\begin{array}{l}\text { CSRMO } \\
\text { criteria }\end{array}$ & $\begin{array}{l}\text { Follow up } \\
\text { (months) }\end{array}$ & $\begin{array}{l}\text { Visual } \\
\text { acuity }\end{array}$ & $\begin{array}{l}\text { CSRMO } \\
\text { criteria }\end{array}$ & \\
\hline 1 & $\mathbf{R}$ & $20 / 200$ & 1 & 5 & $20 / 50$ & 1,3 & 6 & $20 / 50$ & 1,3 & MI, ON, PRP \\
\hline & L & $\mathrm{CF} \ddagger$ & $1,2,3$ & 4 & CF & $1,2,3$ & 65 & $\mathrm{CF}$ & 1,3 & MI, ON, PRP \\
\hline 2 & $\bar{R}$ & $20 / 40$ & $1,2,3$ & 3 & $20 / 30$ & & 40 & $20 / 20$ & None & MI, ON \\
\hline 3 & $\mathrm{~L}$ & $20 / 60$ & $1,2,3$ & 4 & $20 / 50$ & None & 12 & $20 / 50$ & $1,2,3$ & $\mathrm{MI}$ \\
\hline 4 & $\mathbf{R}$ & $20 / 80$ & 1,3 & 3 & $20 / 80$ & 1,3 & 44 & $20 / 200$ & None & MI, ON, PRP, CAT \\
\hline 5 & L & $20 / 100$ & 1,3 & 5 & $20 / 200$ & None & 31 & $20 / 200$ & None & MI, ON, PRP, RTMD \\
\hline כ & L & $20 / 400$ & $\begin{array}{l}1,2 \\
1,3\end{array}$ & 8 & $\begin{array}{l}20 / 40 \\
20 / 200\end{array}$ & 1,2 & $\begin{array}{l}68 \\
76\end{array}$ & $20 / 25$ & None & MI \\
\hline 6 & $\vec{R}$ & CF & $1,2,3$ & 7 & CF & $1,2,3$ & 44 & $20 / 300$ & $\begin{array}{l}\text { None } \\
1,2,3\end{array}$ & MI, ON \\
\hline & $\mathrm{L}$ & $20 / 100$ & $1,2,3$ & 5 & $20 / 100$ & 1,2 & 55 & HM $\|$ & $1,2,3$ & MI, ON, PRP, VH \\
\hline 7 & $\mathbf{R}$ & $20 / 200$ & 1,3 & 7 & $20 / 100$ & 1,3 & 18 & $20 / 50$ & None & MI, PRP, VH, CAT \\
\hline 8 & $\mathbf{R}$ & $20 / 40$ & $1,2,3$ & 4 & $20 / 20$ & $1,2,3$ & 9 & $20 / 20$ & & MI \\
\hline
\end{tabular}

${ }^{\star}$ Criteria are defined in Methods section. +Confounders: $\mathrm{MI}=$ macular ischaemia; $\mathrm{ON}=$ optic neuropathy; $\mathrm{PRP}=$ panretinal photocoagulation; $C A T=$ cataract; $R T M D=$ repaired traction macular detachment; $V H=$ vitreous haemorrhage 


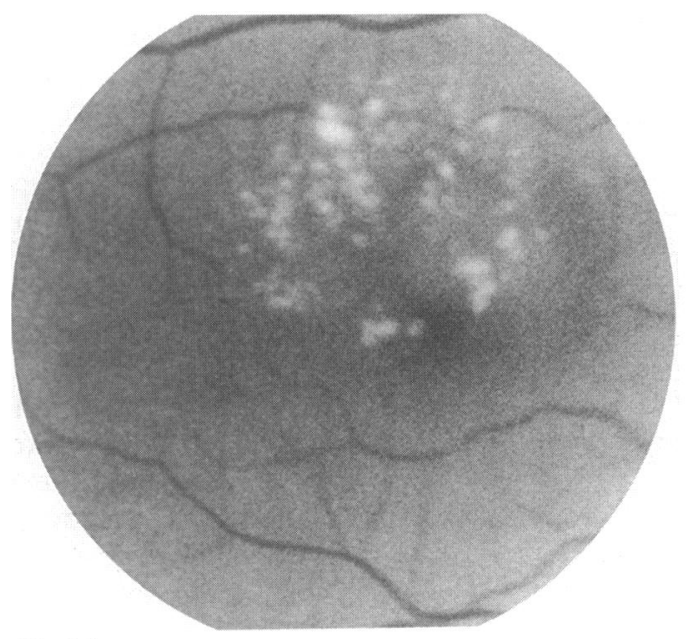

Fig $4 A$

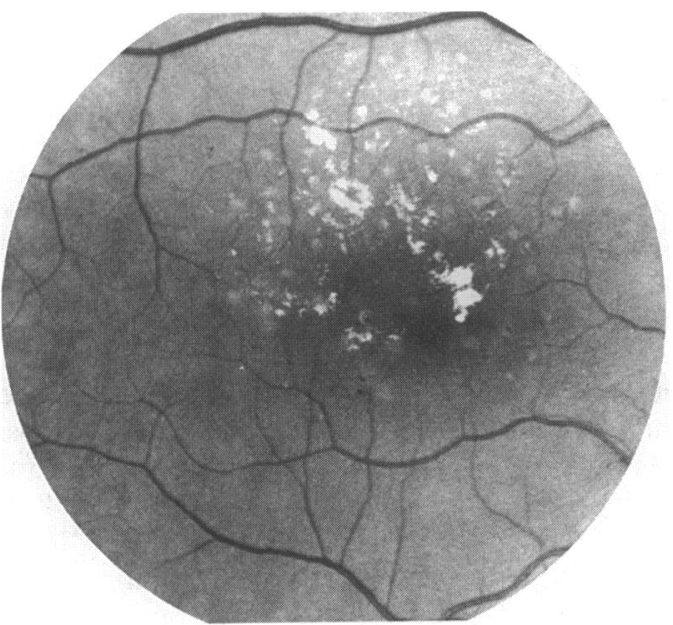

Fig $4 C$

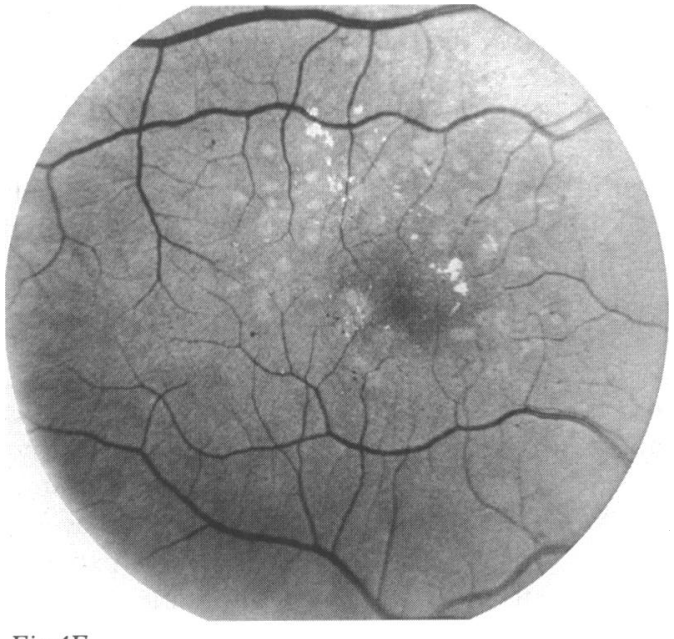

Fig $4 E$

for either diabetic or radiation macular oedema.

Another significant difference between radiation and diabetic retinopathies may be more extensive damage to ciliary and perhaps choroidal vessels (choroidopathy) as well as the retinal pigment epithelium following radiation. ${ }^{10-12}$ The massive exudates seen preoperatively in the centre of the macula in two of our study eyes (cases $1 \mathrm{~L}$ and $6 \mathrm{R}$ ) may be the result of severely compromised transport functions of the retinal pigment epithelium due to radiation damage. Both of these eyes had poor vision pre- and postoperatively.

Optic neuropathy more frequently

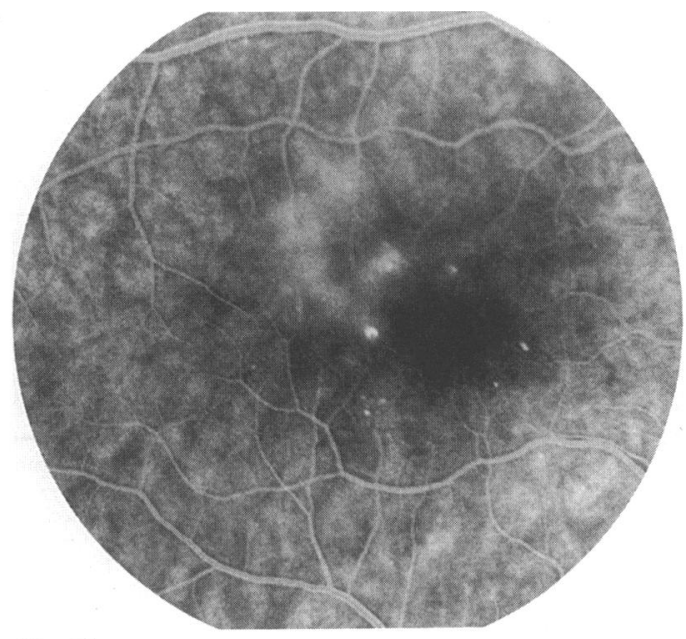

Fig $4 B$

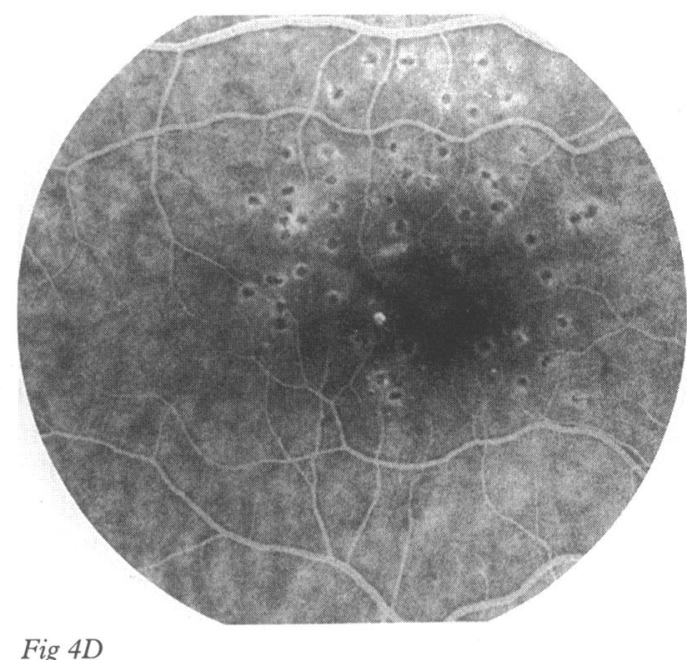

Fig 4D

Figure 4 (A) Preoperative photograph of right macula with all three criteria for CSRMO. (B) Preoperative fluorescein angiogram (late phase) showing

hyperfluorescence of microaneurysms and leakage of dye. (C) Initial postoperative fundus photograph with decreased exudates but persistence of all three CSRMO criteria. (D) Initial postoperative fluorescein angiogram (late phase) showing hypo-and hyperfluorescence of the photocoagulation scars as well as decreased leakage of dye (compared with (B)). (E) Final postoperative fundus photograph shows a further reduction in macular exudates. CSRMO criterion 2 persists, but criteria 1 and 3 are no longer present (compared with $(A)$ and $(C)$ ).

accompanies the diagnosis of radiation retinopathy then diabetic retinopathy. The vessels supplying the optic nerve are probably not as frequently affected by diabetes mellitus as by irradiation. ${ }^{11}$ We believe the pre- and postoperative visual acuities of six of our seven study eyes with optic neuropathy would have been better if the optic nerves had not been adversely affected by the radiation. The other eye (case 2 ) had $20 / 20$ visual acuity postoperatively. The improved vision at the final postoperative examination in this eye and two other eyes (right eye of cases 1 and 6) with optic neuropathy is probably not due to 
spontaneous improvement in vision loss secondary to optic neuropathy, as has been reported, ${ }^{13}$ since the optic neuropathy was diagnosed years before the photocoagulation for CSRMO.

The visual results may have been affected by the panretinal photocoagulation in six study eyes since decreased visual acuity is a known complication of panretinal photocoagulation in eyes with diabetic retinopathy. ${ }^{14}$ Except for the eye with a repaired traction macular detachment, these six eyes had the least chance of having improved vision following macular photocoagulation. The indications for panretinal photocoagulation in these eyes (neovascularisation with preretinal or vitreous haemorrhage designated as radiation retinopathy at high risk for severe vision loss) ${ }^{4}$ suggest that these eyes were more severely affected by the radiation treatment than the eyes not developing these high risk characteristics. The importance of routine, periodic follow up of all patients with radiation retinopathy is emphasised by the vitreous haemorrhage, resultant opacified media, and hand motion visual acuity which occurred more than 4 years after beginning treatment for CSRMO in the left eye of case 6 .

In addition to follow up for development of high risk characteristics, eyes with radiation retinopathy need to be followed for recurrent and persistent CSRMO. Retreatment was offered to our patients if CSRMO was present at any follow up examination and microaneurysms, areas of capillary non-perfusion, or diffuse leakage were identified which could be responsible for the CSRMO. This need for follow up and retreatment, if necessary, is similar to eyes with clinically significant diabetic macular oedema. ${ }^{15}$

In addition to the visual acuity results, we believe the anatomical results also support a beneficial effect of photocoagulation for CSRMO. The anatomical improvement in eight of these 12 eyes, as judged by a decreased number of CSRMO criteria, suggests the treatment techniques used in this study were successful in decreasing the retinal oedema. Not all treated eyes, however, respond as judged by absence or decreased number of CSRMO criteria following treatment. Comparable anatomical results of macular photocoagulation in diabetic eyes are not available for comparison with our data.
In summary, macular photocoagulation for CSRMO shows promise for decreasing vision loss as well as improving vision already lost due to radiation macular oedema. Presently untreatable associated causes for decreased vision (for example, severe macular ischaemia, radiation optic neuropathy, and perhaps choroidopathy with retinal pigment epithelial atrophy) in some patients with CSRMO may prevent or lessen functional improvement (better vision) even though anatomical improvement (decreased oedema) is achieved with macular photocoagulation.

Presented in part at the Annual Meeting of the Association for Research in Vision and Ophthalmology, Sarasota, Florida, May 1993.

Supported in part by NIH Grant EY01730, the Chatlos Foundation (Longwood, FL), and an award from Research to Foundation (Longwood, FL), and
Prevent Blindness, Inc (New York).

1 Guyer DR, Mukai S, Egan KM, Seddon JM, Walsh SM, Gragoudas ES. Radiation maculopathy after proton beam irradiation for choroidal melanoma. Ophthalmology 1992; 99: 1278-85.

2 Packer S, Stoller S, Lesser ML, Mandel FS, Finger PT. Long-term results of iodine 125 irradiation of uveal melanoma. Ophthalmology 1992; 99: 767-74.

3 Collaborative Ocular Melanoma Study. COMS manual of procedures. Springfield, VA: National Technical Information Service; 1989. NTIS Accession No PB90115536.

4 Kinyoun JL, Chittum ME, Wells CG. Photocoagulation treatment of radiation retinopathy. Am $\mathcal{f}$ Ophthalmol 1988; 105: 470-8.

5 Early Treatment Diabetic Retinopathy Study Research Group. Photocoagulation of diabetic macular edema. Arch Ophthalmol 1985; 103: 1796-806.

6 Kinyoun J, Barton F, Fisher M, Hubbard L, Aiello L, Ferris F III, and the ETDRS Research Group. Detection of diabetic macular edema. Ophthalmoscopy versus photography. Ophthalmology 1989; 96: 746-51.

7 Early Treatment Diabetic Retinopathy Study Research Group. Treatment techniques and clinical guidelines for photocoagulation of diabetic macular edema. Ophthalmology 1987; 94: 761-4.

8 Kinyoun JL, Kalina RE, Brower SA, Mills RP, Johnson RH. Radiation retinopathy after orbital irradiation for Graves ophthalmopathy. Arch Ophthalmol 1984; 102: Graves

9 Amoaku WMK, Archer DB. Fluorescein angiographic features, natural course and treatment of radiation retinopathy. Eye 1990; 4: 657-67.

10 Archer DB. Doyne Lecture. Responses of retinal and choroidal vessels to ionising radiation. Eye 1993; 7 $1-13$.

11 Egbert PR, Fajarado LF, Donaldson SS, Moazed K. Posterior ocular abnormalities after irradiation for Posterior ocular abnormalities after irradiation for retinoblastoma: a

12 Hidayat AA, Fine BS. Diabetic choroidopathy. Light and electron microscopic observations of seven cases. Ophthalmology 1985; 92: 512-22.

13 Brown GC, Shields JA, Sanborn G, Augsberger JJ, Savino PJ, Schatz NJ. Radiation optic neuropathy. Ophthalmology 1982; 89: 1489-93.

14 The Diabetic Retinopathy Study Research Group. Photocoagulation treatment of proliferative diabetic retinopathy: the second report of diabetic retinopathy retinopathy: the second report of diabetic reting

15 Early Treatment Diabetic Retinopathy Study Research Group. Photocoagulation for diabetic macular edema. Int Ophthalmol Clin 1987; 27: 265-72. 